\title{
Review
}

\section{The clinical impact of adherence to therapy in airways disease}

For a physician, the final step of a consultation consists of developing a treatment plan and prescription. For the patient, this is the start of a process. First, their role in the treatment plan must be clarified, then they may have to obtain an alternative prescription from their general practitioner. Next, they must have the prescription filled and dispensed from the pharmacy and, finally, they must take the treatment on time and for the required duration. For people with chronic conditions, this requires repeatedly returning to the pharmacy for the prescription to be renewed and dispensed. Given that many patients are on multiple treatment regimens and may have poor health literacy, this becomes a complex process and it is not surprising that this can, and frequently does, go wrong.

Research shows that when a patient does not adhere to standard asthma or COPD treatment, they report poor control and overuse of rescue $\beta$-agonists, experience frequent exacerbations and are often prescribed add-on treatments such as biological agents. In short, poor treatment adherence can manifest in the same way as a refractory condition.

These clinical features should prompt a clinician to investigate poor adherence as they might investigate a new blood or radiological finding. Examining a patient's prescription refill records or a digitally enabled inhaler can demonstrate a number of patterns of inhaler use. A small minority regularly use their treatment as prescribed but many appear to be "cluster users": a group of patients who use their treatment correctly when they are unwell, but once some level of personal control is attained, they cease or reduce their use. Others may cease using their treatment because they are not perceiving a benefit or because an alternative condition accounts for their symptoms. In other words, clinicians can consider that treatment adherence is like a clinical sign: something to be investigated so that they may understand the patient's condition better.

\section{Educational aims}

- To highlight the clinical consequences of poor adherence to standard treatments for airways diseases.

- To describe how poor treatment adherence manifests as complications of the condition.

- To highlight that when a patient does not benefit as might be expected from a treatment, poor adherence should be considered and evaluated for, before more treatment is added.

Cite as: Brennan V, Mulvey C, Costello RW. The clinical impact of adherence to therapy in airways disease. Breathe 2021; 17: 210039. 
Prescribing a treatment is a routine part of a physician's work. Treatment decisions occur after assessing patient symptoms, performing a clinical examination and assessing other relevant diagnostic information, before finally issuing a prescription. For a physician, this is the end of a familiar process. Physicians commonly prescribe the same forms of treatment and this is considered to be good prescribing practice, which makes sense as they are familiar with the benefits, side-effects and dosing schedules. The time available to perform the standard consultation varies from $>20 \mathrm{~min}$ in highly resourced healthcare systems to $<1 \mathrm{~min}$ in some parts of the world [1]. In the rushed setting of a clinic, the time available for a physician to explain the benefits, risks, dosing schedule and how to use a device like an inhaler is compromised.

For the patient being issued the prescription, this is just the beginning of a multistep journey. To adhere to the directions on the prescription, the patient must understand when and how to take the medication. Health literacy is often assumed but rarely tested for and has been directly associated with adherence rates [2]. After receiving their prescription, the patient must then take it to the pharmacy, wait for it to be dispensed, pay for it in some countries, and then take the medication at the directed times over the relevant timeframe. When this happens exactly as the physician intended then the individual is said to have been adherent to the treatment. Given the complexity of the process, it is not surprising that this can go wrong, especially in the setting of chronic disease where the treatment may be lifelong. A patient who does not adhere to asthma or COPD inhaled treatments effectively has an untreated condition, and so develops a number of downstream consequences. This may lead to overuse of inhaled rescue medications in the form of inhaled short-acting $\beta$-agonists (SABAs). For some, insufficient adherence or complete non-adherence to simple inhaled treatments may lead to the prescription of biologics or other add-on treatments, including long-term oral corticosteroids. Studies on treatment adherence are complex, as people do not readily volunteer to being poorly adherent to treatment or may be unaware of their non-adherence (so-called unintentional non-adherence). As a result, most information is gathered from the control limbs of clinical trials or from pharmacies via prescription refill records. Unfortunately, self-reported scales previously used in adherence research are now not encouraged for use, due to recall bias. In this review we outline, from several stakeholders' points of view, the clinical implications that arise when a treatment has not been taken.

\section{Is it just a question that some people do not take their treatment?}

A study evaluating the prevalence of primary non-adherence, a phenomenon where a new medication prescription is never collected, found that only $80 \%$ of patients prescribed a new asthma medication ever collected their prescription [3]. Similarly, a study published by a group in the USA identified that $<10 \%$ of patients prescribed fluticasone propionate/salmeterol refilled their prescription over a 1-year period, a phenomenon called primary non-persistence [4]. This work was performed by reconciliation of prescriptions from an electronic patient record with dispensing records from a pharmacy database. That primary non-adherence and non-persistence exist at all is a surprise to many physicians, much less their prevalence. From a patient's perspective, it is quite reasonable that they would not fill a prescription if they did not believe that the treatment they received is warranted, so called "intelligent nonadherence" or "intentional non-adherence", or if the driver for their original consultation was not addressed. For others, marginalised in society, social issues related to poverty or homelessness mean they cannot easily access the medication [5].
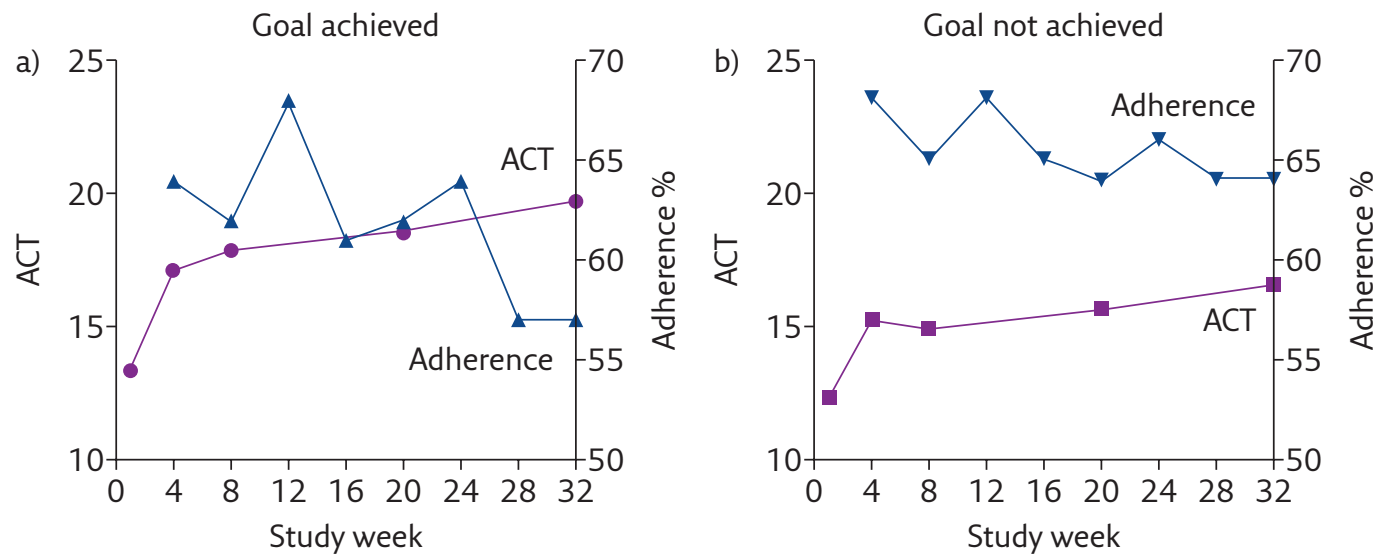

Figure 1 The relationship between ACT scores and adherence in participants who a) did or b) did not achieve their goals. Reproduced and modified from [10] with permission. 
After a medication has been dispensed, it may not be taken or may be taken incorrectly. Overall treatment adherence to inhaled therapy is $~ 50 \%$ when objective tools are used to quantify when and how the inhaler has been used [6-8]. In effect this means that, over a period of time, people receive half of their prescribed doses.

A systematic review of 51 observational studies by Dima et al. [9] corroborated that adherence to inhaled corticosteroids (ICS), a crucial medication in the management of asthma, is $\sim 50 \%$. Dima et al. [9] reported consistent links between adherence rates and the patient's beliefs regarding the inhaler necessity. In other words, a clinician needs to consider the patient's opinion as well as their perspective on the severity of their condition, instead of relying solely on objectively measured values from a standard patient-reported outcome tool, such as the Asthma Control Test (ACT) or Asthma Control Questionnaire (ACQ), because in the real world, patients are likely to adjust their treatment use to what they feel is necessary. In another large cohort of patients with severe asthma, it was noted that nearly half of the treatment goals identified by patients did not align with those deemed important by physicians. It was also noted that, once a patient had achieved their own personal goals for treatment (as opposed to a particular score on the ACT), there was a subsequent decline in adherence to ICS/long-acting $\beta$-agonist (LABA) therapy [10]. This is illustrated in figure 1, which graphs the ACT score and adherence rates (on the $y$-axis) against time. The patients who achieved what they wanted throughout the study had a significant drop in adherence rates over the subsequent period compared to patients who did not achieve their goals.

Stating that people only take half of the prescribed treatment doses does not capture the nuance of how people use their treatment. Some illustrations of the patterns of ICS/LABA use obtained from a digital recording device attached to an inhaler are shown in figures 2-5, to graphically illustrate the issues. The output from this digital device gives an attempted adherence rate and an "actual" adherence rate [11], after dose timing and inhaler technique are accounted for, as well as a time-stamped illustration of ICS/LABA use. For the study [12], patients also underwent peak expiratory flow rate (PEFR) monitoring and the corresponding data for the same period are shown in the lower panels. Figure 2 shows an example of a patient who took their treatment on time and correctly. Among patients with COPD and asthma, this pattern represents $<20 \%$ of patients studied [13]. Figure 3 is an example of a patient who was not regularly adherent to treatment with a twice-daily ICS/LABA. Their use over a period of months shows irregular treatment use and poor control. Figure 4 shows an example of a patient who used their treatment all the time but showed features of refractory disease. Figure 5 shows an example of a patient who misunderstood their physician's instructions on how to use their treatment, ultimately only taking their twice-a-day preparation of ICS/LABA once per day and with poor technique. The patient took treatment on time but suffered a progressive decline in their asthma control.

VAN BOVEN et al. [14] reported data on treatment use from a large database of prescription refills from Australia. They used a novel way of grouping people based on the patterns of adherence to ICS/LABA treatment over time. They identified four treatment trajectories similar to the individual cases discussed earlier: $20 \%$ were nonpersistent in their treatment use, $8 \%$ were seasonal users, $58 \%$ of patients had poor adherence and $13 \%$ had good adherence.

An elegant recent study by DimA et al. [15] prospectively assessed treatment use by 847 patients diagnosed with persistent asthma in France and the UK for 2 years. Using computerassisted telephone interviews, Dıma et al. [15] identified that, at a within-person level, higher than usual ICS adherence was associated with higher contemporaneous reliever use and a subsequent lower inhaler use. In other words, the cyclical use of treatment, probably in response to declining control or asthma attacks, drives adherence to ICS and reliever use. Achieving a reasonable level of control then leads to a decline in reliever use and, at some point, lower ICS use.

A small number of people use their preventer ICS/LABA treatment regularly and on time. Many appear to be cluster users, who use treatment when they are unwell and then, having achieved some level of personal control, cease or reduce their use. Others may cease using their treatment because they are not getting benefit from it. Knowledge of the pattern of inhaler use can give clinicians context when trying to understand a patient's current presentation and may help guide future treatment decisions.

Aside from gaining valuable insights into individual adherence patterns, there is good evidence to show that the use of electronically enhanced inhalers promotes treatment adherence $[6,16,17]$, and recently an integrated smart-inhaler, the Digihaler (TEVA), has received US Food and Drug Administration (FDA) approval. Further research on the effect of these devices on real-world prescribing practice and specialist referrals would be welcomed.

\section{Consequences of poor treatment adherence}

\section{Prescription of more medicines, polypharmacy and associated negative effects}

The Global Initiative for Asthma (GINA) and other international asthma management strategies outline that if a patient is not controlled despite 

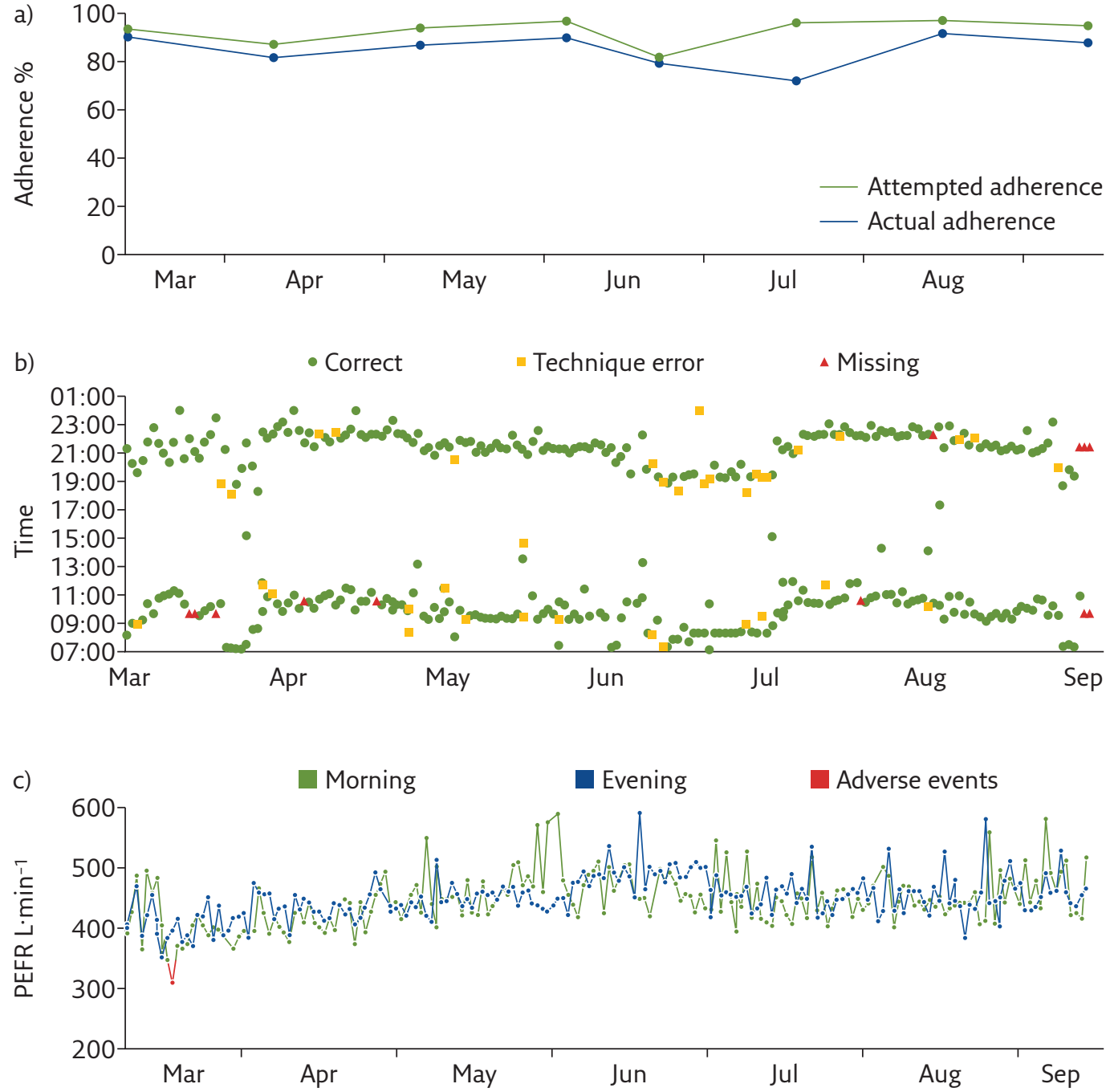

Figure 2 Patient showing good adherence with regular use and stable lung function. a) Attempted and actual adherence. b) ICS/LABA usage pattern indicating mostly good technique, some suboptimal technique and rare missed doses. c) Morning and evening PEFR, also indicating adverse events (exacerbations requiring antibiotics or steroids).

high-dose inhaled ICS/LABA treatment, they should be considered for an add-on therapy. For those who display features of type 2 inflammation, with eosinophilia and/or elevated exhaled nitric oxide fraction, this can be one of the biological agents [18-20]. A problem for clinicians in routine practice is that it can be difficult to identify if a patient is truly refractory to the ICS/LABA or if they have simply not been taking their treatment, something that was highlighted by SULAIMAN et al. [21], who found that only $27 \%$ of patients labelled as having severe uncontrolled asthma were truly refractory when adherence and technique were accounted for.

Several recently published studies have examined this topic. For example, van Boven et al. [14] reported the association between adherence to ICS and step-up to GINA step 5 treatments at a later stage. They identified that nearly $5 \%$ of patients with asthma required escalation to GINA step 5 treatment after ICS/LABA initiation. They also noted that $>80 \%$ were poorly adherent to their inhaled preventer treatments, although poor adherence was associated with longer time to prescription of additional GINA step 5 treatments, suggesting that there was an attempt by the physician to address adherence.

In another recent report, MADDux et al. [22] reported on data from a large database of over half a million asthma patients of whom $>5000$ were prescribed a biological therapy. Using data from pharmacy refills, paid for by a large US insurance company, it was shown that mean adherence to ICS treatment was $<50 \%$ among patients prescribed add-on biological therapy. In a large single-centre retrospective cohort study from the UK by D'ANCONA et al. [23], 18\% of patients had adherence rates to ICS/LABA of $<50 \%$ while on an asthma biological therapy. Furthermore, these people had more exacerbations and less reduction in oral corticosteroid use while on the asthma biological therapy. Hence, there are a lot of emerging data to support the GINA recommendation that adherence to ICS/LABA treatment should be assessed before additional treatment be considered. Clinicians in practice 

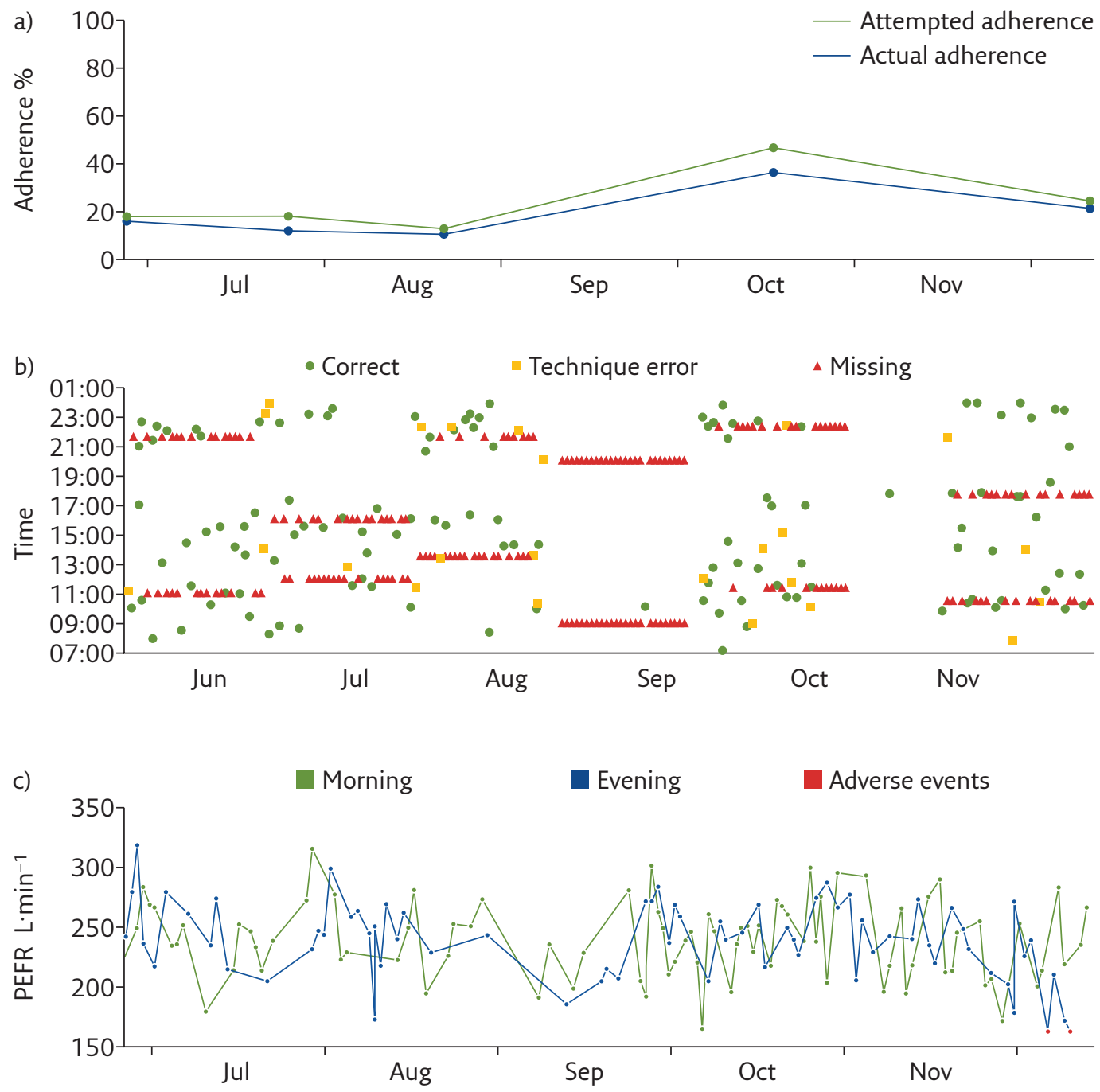

Figure 3 Patient showing poor adherence with irregular use and prolonged periods of no treatment with poor disease control. a) Attempted and actual adherence. b) ICS/LABA usage pattern indicating some good technique, some suboptimal technique and many missed doses. c) Morning and evening PEFR, also indicating adverse events (exacerbations requiring antibiotics or steroids).

should be aware and focus on ensuring adherence to ICS/LABA therapy in this cohort of patients, especially if the expected improvements in disease control are not realised.

In patients with COPD, polypharmacy is increasing. A cross-sectional study performed in the UK found that patients with COPD were more likely to have polypharmacy than patients without, $52 \%$ being on $\geq 5$ regular medications and $15 \%$ being regularly prescribed $\geq 10$ medications [24]. Aside from being associated with high rates of adverse drug reactions [25] and worse physical and cognitive capability [26], the prescription of additional medication in a patient with suboptimal adherence may confound the issue and worsen adherence patterns [27].

\section{Excessive oral corticosteroid and $\beta$-agonist use}

As previously stated, studies examining treatment adherence are quite difficult, especially without the use of specific digital monitoring devices, and therefore often rely on pharmacy refill data. The study by van Boven et al. [14] also showed that almost $50 \%$ of non-adherent patients used two or more courses of oral corticosteroids and antibiotics over the course of the year. The prescription of frequent courses of steroids is common, as there appears to be a perception in the medical community that the benefit of a short burst of steroids is much higher than the risk, especially when used judiciously [28]. That said, taking more than four standard short courses of oral corticosteroid significantly increases the lifetime risk of pneumonia and Cushing syndrome-type sideeffects, including type 2 diabetes, cardiovascular diseases, osteoporosis and cataracts [29].

Similarly, overuse of SABAs is significantly higher among less adherent patients. In a retrospective cohort study in Sweden, over-reliance on SABAs was found in $30 \%$ of patients and was associated with not only an increase in asthma exacerbations, 

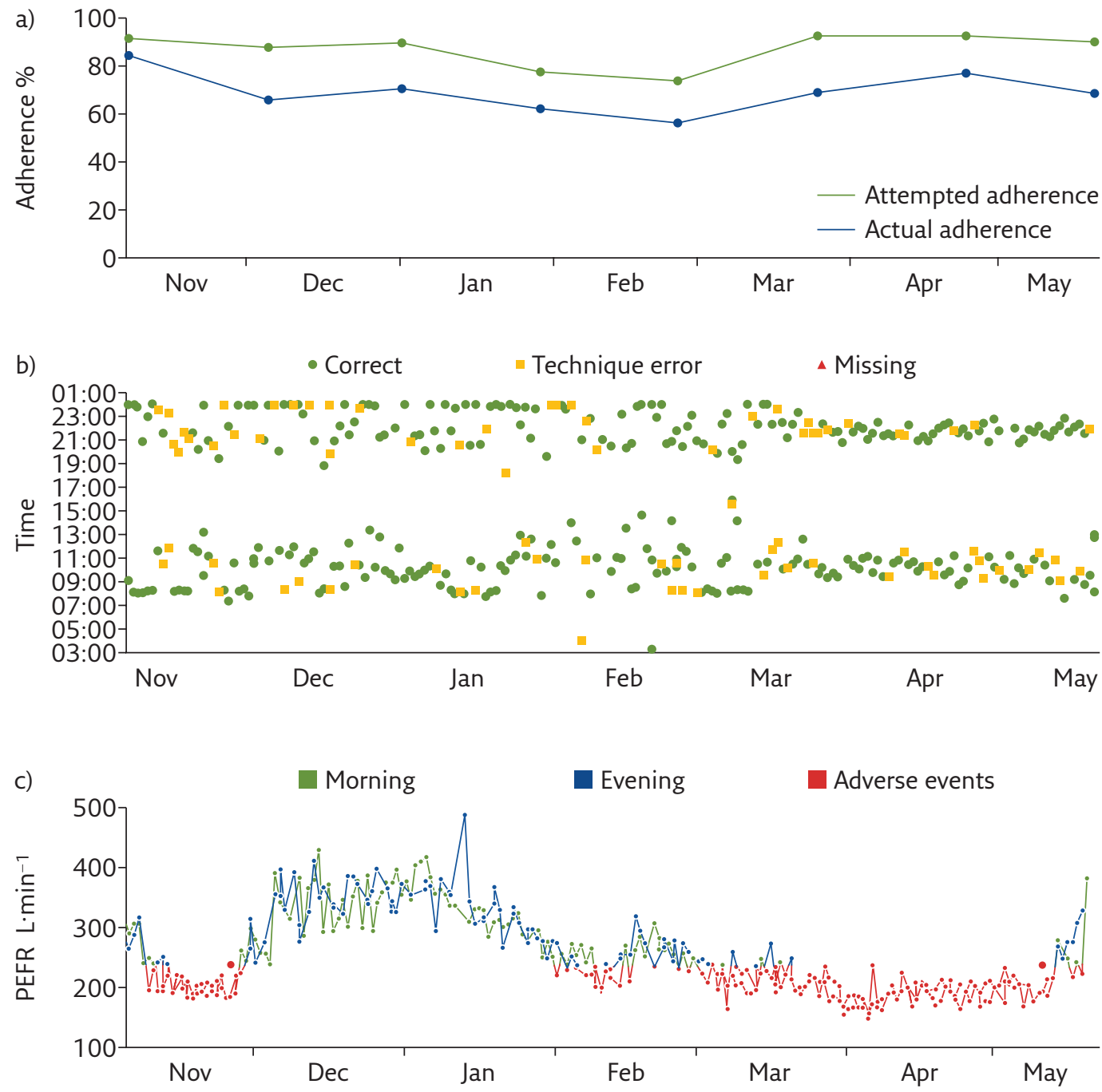

Figure 4 Patient showing good adherence with regular use but refractory disease. a) Attempted and actual adherence. b) ICS/LABA usage pattern indicating mostly good technique, some suboptimal technique and no missed doses. c) Morning and evening PEFR, also indicating many adverse events (exacerbations requiring antibiotics or steroids).

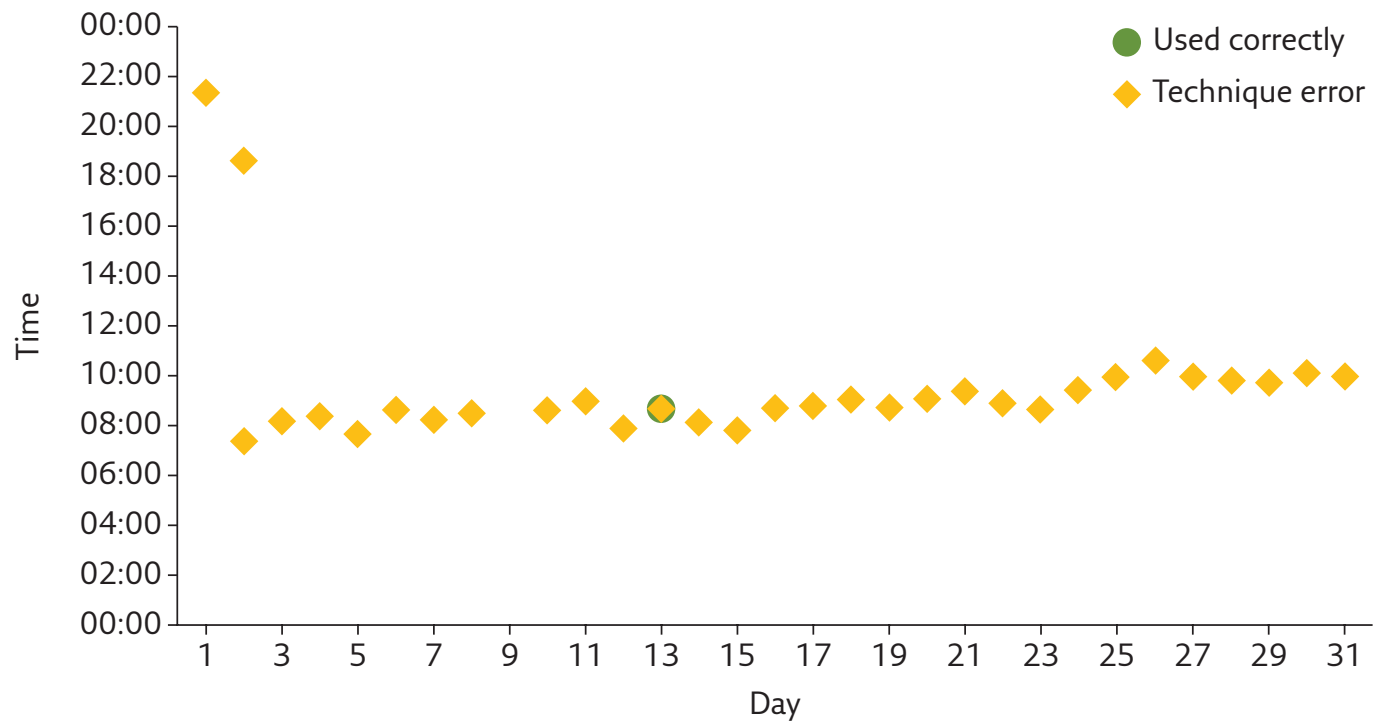

Figure 5 A patient's inhaler use where they took the medication regularly but misunderstood the instructions on when and how to take their inhaler. 
but also an incrementally increased risk of mortality [30]. These findings echo the results of the National Review of Asthma Deaths [31]. The harm of overuse of $\beta$-agonists and underuse of ICS among patients with mild asthma has prompted a revision of the GINA strategy and other guidelines to change their suggested management strategies, which now suggest pulse use of low-dose ICS/formoterol in lieu of SABAs.

\section{Excessive healthcare costs}

For healthcare systems, poor medication adherence leads to downstream costs through complications of partially or incompletely treated conditions. It is estimated that poor medication adherence through the direct effect of untreated conditions and additional add-on treatment costs USD 100-290 billion per year in the USA, with a figure between USD 949 and USD 44190 per person per year for respiratory diseases alone, in 2015 [32]. Most of the costs attributed to nonadherence in patients with COPD are accounted for by hospitalisations and healthcare consultations, which offset the reduced medication costs caused by decreased medication adherence. There are some studies to indicate that targeted interventions on certain cohorts of patients with particular adherence patterns can be cost saving. For example, in a cost modelling study, ZAFARI et al. [33] suggested that a programme that improves adherence by $50 \%$ would result in more upfront costs but fewer exacerbations and more QALYs, therefore increasing the incremental cost-effectiveness ratio. The cost effectiveness of avoiding add-on biological agents and the cost effectiveness of strategies to avoid multiple courses of oral corticosteroids are yet to be investigated.

\section{Excessive or inappropriate ICS exposure}

For some patients with symptoms of airways diseases, prolonged use of anti-inflammatory corticosteroids will lead to side-effects, in particular adrenal gland suppression and Cushing syndrome-like complications such as osteoporosis, diabetes and cataracts. While there has been a paradigm shift in the management of COPD, with guidelines suggesting treatment with longacting bronchodilators alone or in combination, there remains a significant proportion of patients prescribed regimes including ICS [34, 35]. Given the lack of easily accessible diagnostic pathways, many cases of physician-diagnosed asthma have been shown to have no evidence of active airway inflammation or hyperreactivity [36] and the clinical challenge going forward in trying to mitigate these unwanted and morbid side-effects is knowing when to down-titrate the dose or even stop these treatments.

\section{Self-evaluation questions}

1. What is primary non-adherence?
a) Not taking medication prescribed in primary care
b) Not taking a medication that is felt to be of no benefit
c) Not collecting prescriptions from the pharmacy
d) Forgetting to take your medication

2. What percentage of patients on biological therapy have an adherence rate to ICS/LABA of $<50 \%$ ?
a) $<5 \%$
b) $5-10 \%$
c) $10-15 \%$
d) $15-20 \%$

3. How does non-adherence manifest clinically?

a) Non-adherence has the same clinical presentation as uncontrolled disease

b) The patient will tell you that they are not taking their treatment

c) You will detect non-adherence if you use a scoring system like the ACT

d) The patient's pharmacist will call to inform you

4. Which of the following is a potential adverse effect of poor adherence?
a) Adrenal insufficiency
b) Cushing syndrome
c) Pneumonia
d) All of the above

\section{Association is not causation: when poor treatment adherence is a clinical sign}

The association between poor adherence and excessive use of short-term oral corticosteroids, reliever bronchodilators as well as add-on therapy is plausibly due to not treating the underlying condition. However, unless the drivers of poor adherence are addressed, non-adherence is likely to remain a recalcitrant medical conundrum. As physicians, we cannot underestimate how hard it is to complete the sequence of getting a prescription, turning it in at the pharmacy, collecting the medication and taking it correctly and on time. It is easy to see how people with poor social support, difficulties in transport, poor literacy or cognitive impairment would struggle to complete this sequence. It should not therefore be a surprise that some of the association of excessive unscheduled healthcare with poor medication adherence arises because it also reflects the individual's psychological and social circumstances.

Unfortunately, it is rare that a physician is truly aware of how and when their patients have used their prescribed treatment. With the current time restraints faced by most physicians, finding the time to obtain all the necessary data that would allow them to identify poor treatment adherence is difficult. While the advent of centralised electronic 
prescribing databases in some countries may make this easier, these are not yet ubiquitous and reviewing pharmacy refills is time consuming, as indeed is the use of adherence monitoring devices, although their potential in future respiratory care is promising [37]. Increased ease of access to these data will provide an opportunity to incorporate adherence knowledge into clinical workflow, rather than adding to the patient burden of treatment when poor adherence is contributing to poor outcomes. Addressing poor adherence may change the dynamic of the doctor-patient relationship but the use of patient-centred, non-judgemental communication skills can unmask previously unidentified barriers to adherence and result in better patient care.

\section{Key points}

- Suspected poor treatment adherence should be considered in the same way as a clinical sign and is something that needs to be investigated.

- Poor treatment adherence should be suspected among people with asthma and COPD who have excessive reliever use and recurrent exacerbations.

- Poor treatment adherence can be detected by assessing medication refill records or using digital devices.

- Prior treatment adherence should be assessed when people are being considered for add-on therapy.

Affiliations

\section{Vincent Brennan ${ }^{1,2}$, Christopher Mulvey², Richard W. Costello ${ }^{1,2}$}

${ }^{1}$ Beaumont Hospital, Dublin, Ireland. ${ }^{2}$ INCA Group, Dept of Medicine, Royal College of Surgeons in Ireland, Dublin, Ireland.

\section{Conflict of interest}

V. Brennan has nothing to disclose. C. Mulvey has nothing to disclose. R.W. Costello reports grants and speaker fees from Aerogen and GSK, and speaker fees from Boehringer Ingelheim, Novartis and TEVA, outside the submitted work. In addition, R.W. Costello has a licensed patent: EP12182189.6 "System and Method for Monitoring Use of a Device".

\section{References}

1. Irving G, Neves AL, Dambha-Miller $\mathrm{H}$, et al. International variations in primary care physician consultation time: a systematic review of 67 countries. BMJ Open 2017; 7 e017902.

2. King A. Poor health literacy: a "hidden" risk factor. Nat Rev Cardiol 2010; 7: 473-474.

3. Fischer MA, Stedman MR, Lii J, et al. Primary medication nonadherence: analysis of 195,930 electronic prescriptions. J Gen Intern Med 2010; 25: 284-290.

4. Bender BG, Pedan A, Varasteh LT. Adherence and persistence with fluticasone propionate/salmeterol combination therapy. J Allergy Clin Immunol 2006; 118: 899-904.

5. Williams LK, Joseph CL, Peterson EL, et al. Race-ethnicity, crime, and other factors associated with adherence to inhaled corticosteroids. J Allergy Clin Immunol 2007; 119: 168-175.

6. Morton RW, Elphick HE, Rigby AS, et al. STAAR: a randomised controlled trial of electronic adherence monitoring with reminder alarms and feedback to improve clinical outcomes for children with asthma. Thorax 2017; 72: 347-354.

7. Foster JM, Usherwood T, Smith L, et al. Inhaler reminders improve adherence with controller treatment in primary care patients with asthma. J Allergy Clin Immunol 2014; 134 1260-1268

8. O'Dwyer S, Greene G, MacHale E, et al. Personalized biofeedback on inhaler adherence and technique by community pharmacists: a cluster randomized clinical trial. J Allergy Clin Immunol Pract 2020; 8: 635-644.

9. Dima AL, Hernandez G, Cunillera O, et al. Asthma inhaler adherence determinants in adults: systematic review of observational data. Eur Respir J 2015; 45: 994-1018.

10. Mulvey C, MacHale E, Greene G, et al. Patient-selected treatment goals in severe asthma. J Allergy Clin Immunol Pract 2021; in press [https://doi.org/10.1016/j.jaip.2021.01.041].

11. Sulaiman I, Seheult J, MacHale E, et al. A method to calculate adherence to inhaled therapy that reflects the changes in clinical features of asthma. Ann Am Thorac Soc 2016; 13: 1894-1903.

12. Mokoka MC, Lombard L, MacHale EM, et al. In patients with severe uncontrolled asthma, does knowledge of adherence and inhaler technique using electronic monitoring improve clinical decision making? A protocol for a randomised controlled trial. BMJ Open 2017; 7: e015367. 
13. Sulaiman I, SeheultJ, MacHale E, et al. Irregular and ineffective: a quantitative observational study of the time and technique of inhaler use. J Allergy Clin Immunol Pract 2016; 4: 900-909.

14. van Boven JFM, Koponen M, Lalic S, et al. Trajectory analyses of adherence patterns in a real-life moderate to severe asthma population. J Allergy Clin Immunol Pract 2020; 8: 1961-1969.

15. Dima AL, van Ganse E, Stadler G, et al. Does adherence to inhaled corticosteroids predict asthma-related outcomes over time? A cohort study. Eur Respir J 2019; 54: 1900901.

16. Chan AHY, Stewart AW, Harrison J, et al. The effect of an electronic monitoring device with audiovisual reminder function on adherence to inhaled corticosteroids and school attendance in children with asthma: a randomised controlled trial. Lancet Respir Med 2015; 3: 210-219.

17. Moore A, Preece A, Sharma R, et al. A randomised controlled trial of the effect of a connected inhaler system on medication adherence in uncontrolled asthmatic patients. Eur Respir J 2021; 57: 2003103

18. Ortega HG, Liu MC, Pavord ID, et al. Mepolizumab treatment in patients with severe eosinophilic asthma. $N$ Engl J Med 2014; 371: 1198-1207.

19. Castro M, Zangrilli J, Wechsler ME, et al. Reslizumab for inadequately controlled asthma with elevated blood eosinophil counts: results from two multicentre, parallel, double-blind, randomised, placebo-controlled, phase 3 trials. Lancet Respir Med 2015; 3: 355-366.

20. FitzGerald JM, Bleecker ER, Nair P, et al. Benralizumab, an anti-interleukin -5 receptor $\alpha$ monoclonal antibody, as add-on treatment for patients with severe, uncontrolled, eosinophilic asthma (CALIMA): a randomised, doubleblind, placebo-controlled phase 3 trial. Lancet 2016; 388 : 2128-2141.

21. Sulaiman I, Greene G, MacHale E, et al. A randomised clinical trial of feedback on inhaler adherence and technique in patients with severe uncontrolled asthma. Eur RespirJ 2018. 51: 1701126.

22. Maddux JT, Inselman JW, Jeffery MM, et al. Adherence to asthma biologics: implications for patient selection, step therapy, and outcomes. Chest 2021; 159: 924-932

23. d'Ancona G, Kavanagh J, Roxas C, et al. Adherence to corticosteroids and clinical outcomes in mepolizumab therapy for severe asthma. Eur Respir J 2020; 55: 1902259.

24. Hanlon P, Nicholl BI, Jani BD, et al. Examining patterns of multimorbidity, polypharmacy and risk of adverse drug reactions in chronic obstructive pulmonary disease: a cross-sectional UK Biobank study. BMJ Open 2018; 8: e018404.

25. Hajjar ER, Hanlon JT, Artz MB, et al. Adverse drug reaction risk factors in older outpatients. Am J Geriatr Pharmacother 2003; 1: 82-89.

26. Rawle MJ, Cooper R, Kuh D, et al. Associations between polypharmacy and cognitive and physical capability: a British birth cohort study. J Am Geriatr Soc 2018; 66: 916-923.

27. Eriksen CU, Kyriakidis S, Christensen LD, et al. Medicationrelated experiences of patients with polypharmacy: a systematic review of qualitative studies. BMJ Open 2020; 10: e036158.

28. Price D, Castro M, Bourdin A, et al. Short-course systemic corticosteroids in asthma: striking the balance between efficacy and safety. Eur Respir Rev 2020; 29: 190151.

29. Price DB, Trudo F, Voorham J, et al. Adverse outcomes from initiation of systemic corticosteroids for asthma: long-term observational study. J Asthma Allergy 2018; 11: 193-204.

30. Nwaru BI, Ekström M, Hasvold P, et al. Overuse of short-acting $\beta_{2}$-agonists in asthma is associated with increased risk of exacerbation and mortality: a nationwide cohort study of the global SABINA programme. Eur RespirJ 2020; 55: 1901872.

31. Levy ML. The national review of asthma deaths: what did we learn and what needs to change? Breathe 2015; 11: 14-24.

32. Cutler RL, Fernandez-Llimos F, Frommer M, et al. Economic impact of medication non-adherence by disease groups: a systematic review. BMJ Open 2018; 8: e016982.

33. Zafari Z, Lynd LD, FitzGerald JM, et al. Economic and health effect of full adherence to controller therapy in adults with uncontrolled asthma: a simulation study. J Allergy Clin Immunol 2014; 134: 908-915.

34. Koblizek V, Milenkovic B, Barczyk A, et al. Phenotypes of COPD patients with a smoking history in Central and Eastern Europe: the POPE Study. Eur RespirJ 2017; 49: 1601446.

35. Ghosh S, Anderson WH, Putcha N, et al. Alignment of inhaled chronic obstructive pulmonary disease therapies with published strategies. Analysis of the Global Initiative for Chronic Obstructive Lung Disease recommendations in SPIROMICS. Ann Am Thorac Soc 2019; 16: 200-208.

36. Aaron SD, Vandemheen KL, FitzGerald JM, et al. Reevaluation of diagnosis in adults with physician-diagnosed asthma. JAMA 2017; 317: 269-279.

37. Blakey JD, Bender BG, Dima AL, et al. Digital technologies and adherence in respiratory diseases: the road ahead. Eur Respir J 2018; 52: 1801147

\section{Suggested answers}

1. c.

2. d.

3. a.

4. d. 